\title{
Géolinguistique
}

19 | 2019

Varia

\section{Atlanti interpretativi, cartografia sintetica, distanza linguistica. Il banco di prova dell'APV- Atlas des patois valdôtains}

Atlas interprétatif, cartographie synthétique, distance linguistique. Le banc d'essai de l'APV-Atlas des patois valdôtains

Interpretative Atlas, Synthetic Cartography, Linguistic Distance. The Test Bench of the APV-Atlas des Patois Valdôtains

\section{Gianmario Raimondi}

\section{OpenEdition}

\section{Journals}

Edizione digitale

URL: http://journals.openedition.org/geolinguistique/1170

DOI: $10.4000 /$ geolinguistique. 1170

ISSN: 2650-8176

Editore

UGA Éditions/Université Grenoble Alpes

\section{Edizione cartacea}

ISBN: 978-2-37747-166-9

ISSN: 0761-9081

Notizia bibliografica digitale

Gianmario Raimondi, « Atlanti interpretativi, cartografia sintetica, distanza linguistica. II banco di prova dell'APV-Atlas des patois valdôtains », Géolinguistique [Online], 19 | 2019, online dal 09 décembre 2019, consultato il 07 octobre 2020. URL : http://journals.openedition.org/geolinguistique/1170; DOI :

https://doi.org/10.4000/geolinguistique.1170

Questo documento è stato generato automaticamente il 7 octobre 2020.

Géolinguistique 


\section{Atlanti interpretativi, cartografia sintetica, distanza linguistica. Il banco di prova dell'APV-Atlas des patois valdôtains}

Atlas interprétatif, cartographie synthétique, distance linguistique. Le banc

d'essai de l'APV-Atlas des patois valdôtains

Interpretative Atlas, Synthetic Cartography, Linguistic Distance. The Test Bench

of the APV-Atlas des Patois Valdôtains

Gianmario Raimondi

\section{L'APV-Atlas des patois valdôtains}

1 L'APV-Atlas des patois valdôtains è un progetto di atlante regionale scaturito dal dibattito geolinguistico degli anni Sessanta-Settanta del Novecento che portò, soprattutto in Francia (Dauzat, 1955) e conseguentemente in Italia, alla creazione dei cosiddetti «atlanti di seconda generazione» (Cugno \& Massobrio, 2010: 205).

Sotto l'egida dell'Assessorato alla Cultura della Regione Autonoma della Valle d'Aosta, la raccolta dei dati cominciò all'inizio degli anni Settanta, con inchieste sul campo (basate sul Questionnaire pour enquête dialectale en pays alpin elaborato in Tuaillon, 1972-1973) su una rete di sedici punti d'inchiesta valdostani, distribuiti sul territorio della regione e rilevanti in rapporto alla sua configurazione geografica, cui vennero aggiunti un certo numero di punti esterni di confronto con le aree francoprovenzali confinanti: il Vallese svizzero, la Savoia e l'Alta Savoia, l'area francoprovenzale piemontese (fig. 1) ${ }^{1}$. 
Figura 1. - La Valle d'Aosta, con alcuni elementi geografici di orientamento, e i punti d'inchiesta APV (v. nota 1).

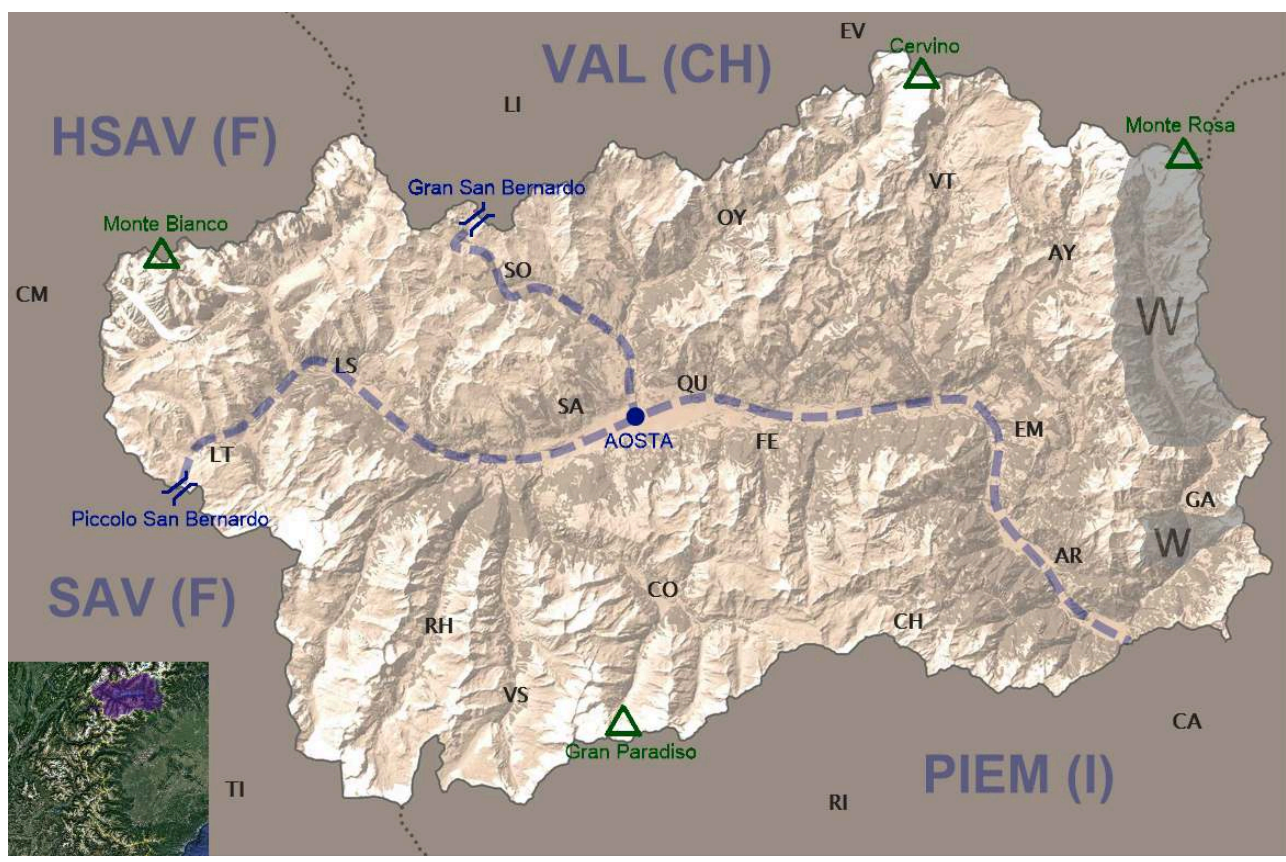

Dopo un lungo periodo in cui i progressi del lavoro si sono alternati a fasi di difficoltà e di stallo², a partire dal 2014 i lavori dell'atlante sono ripresi sotto la co-direzione di Saverio Favre (Regione Autonoma Valle d'Aosta) e Gianmario Raimondi (Università della Valle d'Aosta), in vista della revisione dei materiali e della progettazione del formato editoriale del volume-pilota dell'atlante, la cui pubblicazione è prevista entro il 2020.

4 Proprio a partire dalla veste che i materiali linguistici dell'atlante assumeranno in questo volume-pilota dell'APV, che sarà dedicato alle pratiche connesse al latte e alla caseificazione (Le lait et les activités laitières), in questo contributo illustreremo alcuni aspetti della sua struttura informativa e proporremo alcune riflessioni metodologiche, relative in particolare alla cartografia sintetica utilizzata e al possibile utilizzo di questa in chiave dialettometrica.

\section{2. «Dato» e «interpretazione del dato» nella struttura informativa dell'APV}

Anche per le ragioni connesse al grande lasso di tempo che intercorre fra il momento della raccolta dei dati APV e la loro pubblicazione, nel momento in cui si è cominciato a ragionare sulla pubblicazione l'opzione che è apparsa obbligatoria è stata quella di proporre un atlante «interpretativo», che non si limitasse cioè a fornire i dati secondo una prospettiva puramente classificatoria e descrittiva ma li proponesse già corredati di strumenti per la loro lettura.

6 Questa politica editoriale, oltre che da ragioni epistemologiche e scientifiche da tempo espresse dal pensiero geolinguistico (fra gli altri Alinei, 1994: 23), è motivata anche da un'esigenza di «disseminazione» del sapere dialettologico presso un pubblico che, per espressa volontà dell'Assessorato regionale, non può essere composto solo da specialisti e che necessita quindi di una opportuna «mediazione didattica», e si configura quindi 
anche come una doverosa «restituzione» dei risultati di una ricerca ormai cinquantennale alla comunità dei parlanti francoprovenzali valdostani (Raimondi, 2017: 28-29).

7 Sulla base di tali premesse metodologiche, il progetto di pubblicazione dell'APV (per maggiori dettagli sul quale, accompagnati da illustrazioni, si rimanda nuovamente a Raimondi, 2019) si è via via indirizzato verso una significativa «mediazione interpretativa» a vantaggio del lettore che però preservasse anche l'autenticità dei materiali originari, attraverso una distinzione anche editoriale fra «dato» e «interpretazione del dato».

Figura 2. - Un esempio di Article APV [APV 1-37 le grand bidon à dos (pour lait)].

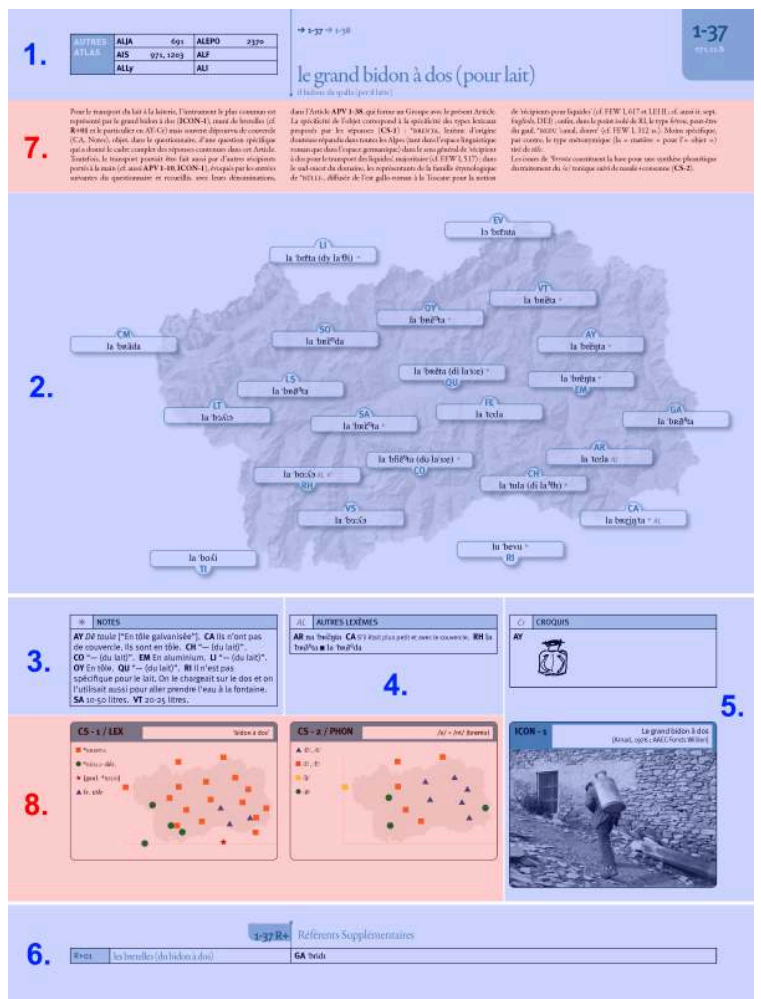

Nelle voci di cui è composto l'atlante (Articles, di cui si fornisce in fig. 2 un esempio standard) l'esposizione ordinata del «dato» è affidata a una serie di elementi informativi (riquadrati e numerati in blu nella fig. 2) rappresentati da: 1 . Intestazione e informazioni di corredo; 2. Carta analitica delle risposte; 3. Note degli informatori; 4. Eventuali varianti fonetiche e morfologiche delle risposte in carta o seconde risposte; 5. Iconografia; 6. Eventuali referenti aggiuntivi rispetto all'intestazione principale della carta, derivanti dalla trasformazione in lemma di informazioni supplementari fornite degli informatori durante le inchieste, e presentati in forma di carta ridotta $\left(C_{+}\right)$oppure di semplice lista di risposte $\left(R+\right.$, se attestate in meno di sette punti d'inchiesta) ${ }^{3}$.

Gli elementi di «interpretazione del dato» (in rosso) sono invece costituiti dalla coppia 7. Commento e 8. Carte sintetiche. I Commenti sono strutturati in un formato piuttosto standard che prevede in linea generale una prima parte in cui vengono presentati gli aspetti relativi alla resa della/e domanda/e del questionario processata/e nella voce (comprensione dello stimolo, conoscenza del referente proposto, eventuali discrepanze fra quadro referenziale proposto e «enciclopedia» o «tassonomie» cognitive del 
parlante) e alla conseguente organizzazione informativa della voce (eventuali riformulazioni del titolo, ridistribuzione dell'informazione, presenza di referenti supplementari, rimandi all'iconografia disponibile), una seconda parte invece dedicata al commento dei dati, comprendente osservazioni e proposte di lettura di carattere sia analitico (sui piani storico-linguistico ed etimologico, fonologico e morfosintattico, etnologico), sia di sintesi geolinguistica in relazione alle configurazioni spaziali emergenti, se degne di nota, istituendo un rapporto dialettico continuo con le Carte sintetico-simboliche (CS) predisposte e pubblicate a completamento delle voci.

L'insieme delle CS raggiunge il numero ragguardevole di 393 unità e comprende anche sintesi riferite ad aspetti etnologici (CS ETHN: 23; cfr. fig. 3) ${ }^{4}$. La maggior parte di esse (370) rappresenta però la distribuzione geografica di fatti eminentemente linguistici, riferiti soprattutto al livello fonetico (CS PHON: 229) e lessicale (CS LEX: 164) $\mathrm{e}$, in misura minore data la focalizzazione tradizionalmente lessicale del questionario, a quello morfo-sintattico (CS MORPH: 20). All'interno del gruppo delle CS lessicali, oltre alle consuete sintesi onomasiologiche, figura inoltre anche un certo numero di carte di carattere semasiologico («cosa significa X [dove X = significante]», CS LEX-S: 10) e semantico-motivazionale («quale iconimo sta alla base dell'adozione diX per $Y$ [dove $\mathrm{X}=$ significante e $\mathrm{Y}=$ referente]», CS LEX-M: 19) ${ }^{5}$. Per tutte le carte linguistiche, infine, sono possibili realizzazioni di sintesi comparative fra due fenomeni.

Figura 3. - Un esempio di sintesi etnografica [da APV 1-67 le moule à beurre; v. nota 5].

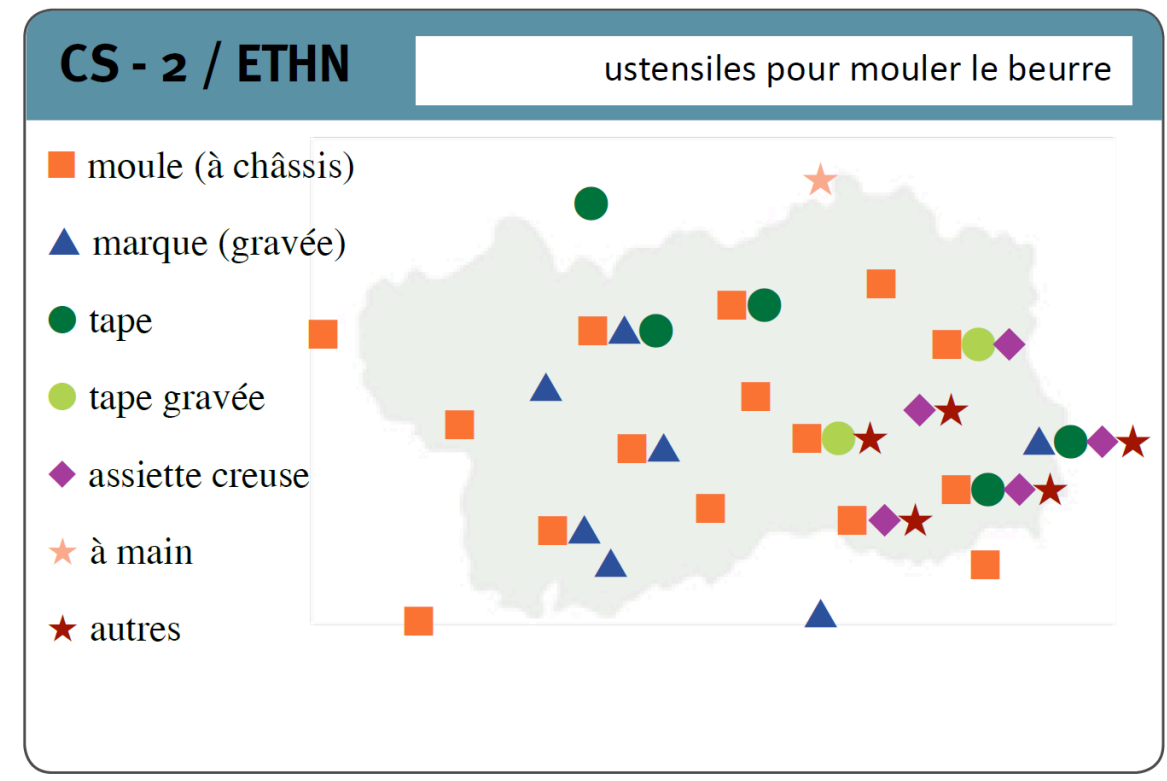

\section{La cartografia sintetica nell'APV come «interpretazione del dato»}

11 Se ogni operazione di classificazione linguistica è di fatto un processo di «interpretazione del dato» (assunto che crediamo sia condivisibile da qualunque linguista, senza ulteriori dimostrazioni), allora il lavoro piuttosto impegnativo compiuto durante la classificazione e la tipizzazione etimologica, grammaticale e fonologica dei dati lessicali, morfosintattici e fonetici del futuro primo volume dell'APV 
in vista della redazione delle sue 370 sintesi simboliche, ha rappresentato senz'altro uno sforzo notevole di tipo interpretativo. Qui di seguito forniamo alcuni esempi di ambito lessicale e fonologico tratti dai materiali in fase di lavorazione avanzata dell'APV (3.2 e 3.3), chiarendo la metodologia adottata e, al contempo, prefigurando alcuni dei risultati (3.4) che è parso interessante sottoporre al banco di prova della dialettometria.

\subsection{Simbologia «semantica»}

12 Alla classificazione linguistica, la metodologia adottata nell'atlante ha abbinato la ricerca di una simbolizzazione grafica capace di rappresentare efficacemente le tipizzazioni compiute ai diversi livelli di analisi dei dati, utilizzando i due livelli iconici dei simboli adottati, ovvero le «forme» e $\mathrm{i}$ "colori», in maniera semanticamente significativa. Le cinque «forme/colore» adottate (quadrato/arancio, rombo/viola, triangolo/blu, cerchio/verde e stella/bruno) rappresentano quindi generalmente snodi tassonomici di livello superiore (ad esempio tipi lessicali coetimologici, oppure esiti fonetici per qualche ragione assimilabili), articolati in manifestazioni diversificate rappresentate dalle diverse tre sfumature (basate principalmente sulla luminosità) della gamma cromatica abbinata.

Alcune delle cinque forme/colore sono inoltre di regola abbinate a determinate configurazioni di distribuzione geolinguistica. In particolare, il quadrato/arancio è utilizzato per i tipi linguistici maggioritari oppure per quelli rappresentati nella cosiddetta «Alta Valle» (parte occidentale della regione) in opposizione al triangolo/blu che identifica quelle della «Bassa Valle», mentre il rombo/viola spesso rappresenta tipi definibili come «intermedi» rispetto a quelli rappresentati dai due simboli precedenti; il cerchio/verde è abbinato spesso a tipi linguistici minoritari dell'area valdostana; infine, la stella/bruno rappresenta di regola tipi isolati oppure attestati solo nell'area francoprovenzale non italiana.

\subsection{Classificazione linguistica: il lessico}

Le sintesi di tipo lessicale sono state realizzate a partire da una tipizzazione etimologica di cui si rende puntualmente conto nei commenti, il cui risultato si può vedere nell'esempio in fig. 4 («pis de la vache») all'interno di una sintesi di carattere onomasiologico dei contenuti della carta analitica riportata sopra: il lessotipo dominante nell'Alta Valle, dalla base forse latina, forse germanica *titta (DEI, tetta e FEW XVII, 334), nelle sue due varianti centrale (con suffissazione in -inu) e occidentale (con altro suffisso) evidenziate dalle sfumature del simbolo quadrato/arancio; i lessotipi concorrenti nell'area regionale, e cioè i continuatori degli etimi lat. pěctu (sul modello anche semantico del francese pis: cerchio/verde), diffuso nella metà orientale, e tardo-lat. *pŭppa (DELI, póppa ${ }^{1}$ : triangolo/blu), soprattutto nei punti più a contatto col piemontese; infine, nel Vallese e in Alta Savoia, l'altro tipo latino, forse più arcaico, *uberu (metaplasmo sul lat. class. ūber, -ris; FEW XIV, 1: stella/bruno). 
Figura 4. - Un esempio di realizzazione di una sintesi lessicale «onomasiologica», da APV 1-5 le pis (de la vache).

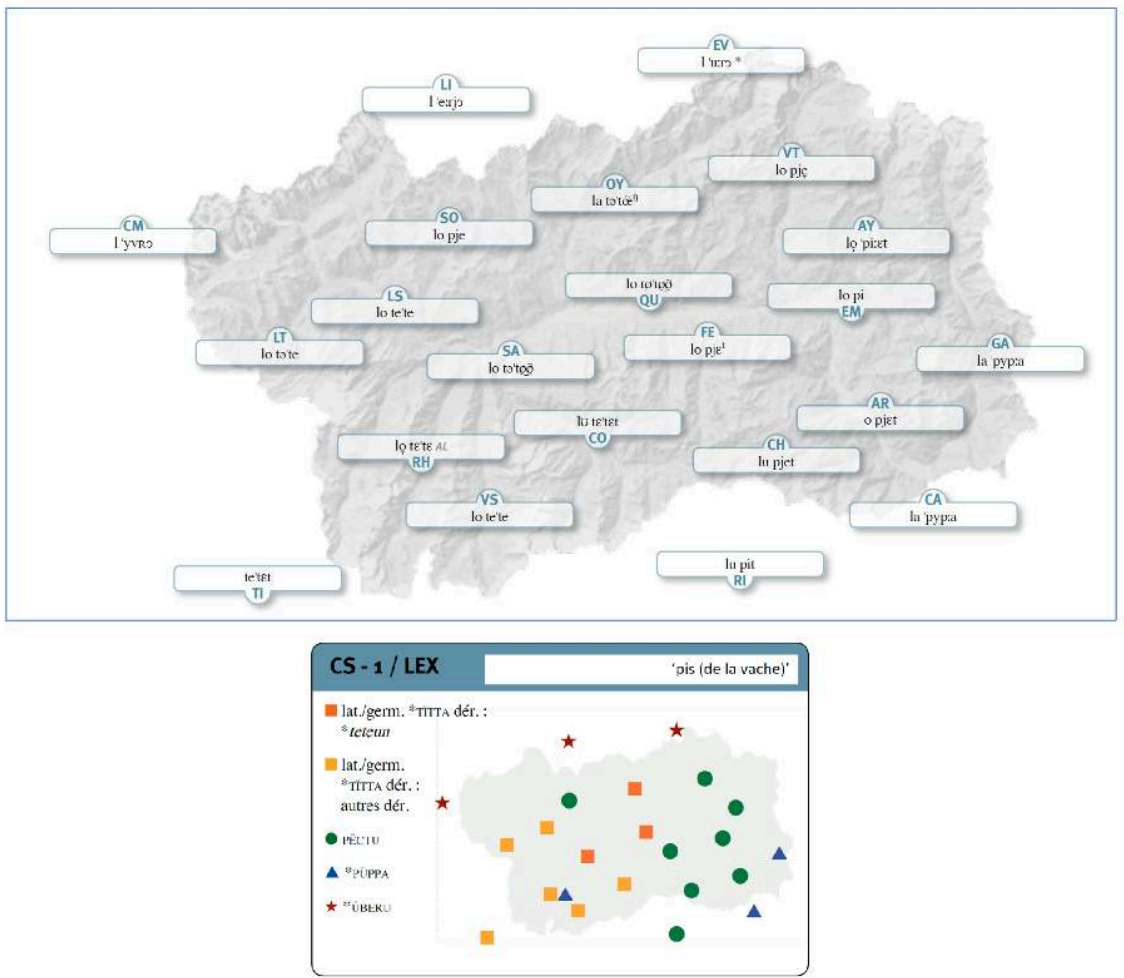

La stessa voce APV permette di esemplificare anche due sintesi lessicali di tipo invece semasiologico (LEX-S, fig.5), dedicate al duplice significato di «mammella» e «capezzolo» (con dati comparativi tratti dalla voce successiva APV 1-6 un trayon/un capezzolo) che sia i continuatori di *tĭtta (CS-2) che quelli di * pŭppa (CS-3) possono assumere a seconda delle aree, anche in questo caso significative dal punto di vista geolinguistico, come mostra la distribuzione dei simboli.

Figura 5. - Due sintesi lessicali «semasiologiche», da APV 1-5 le pis (de la vache).
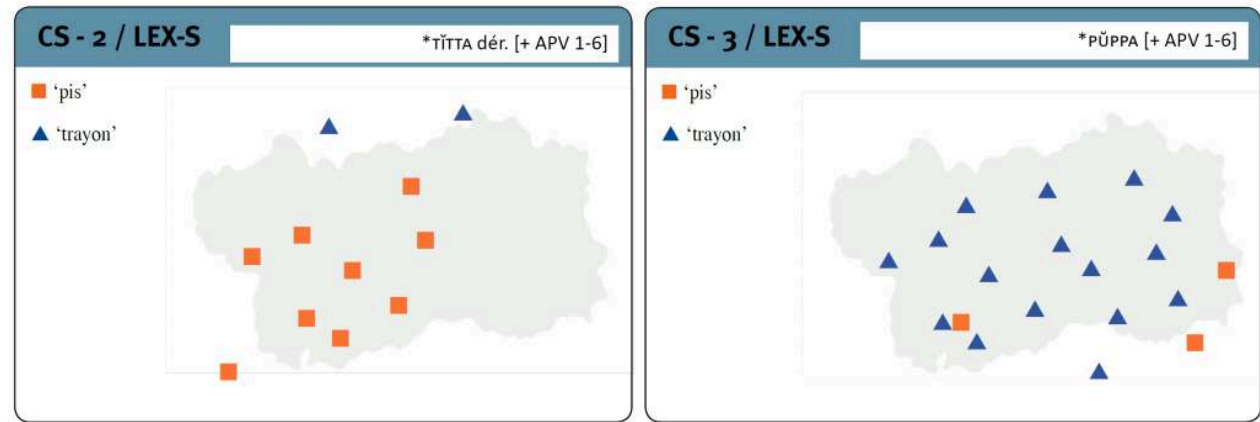

L'esempio successivo (fig. 6) mostra invece, insieme alla relativa carta analitica completa del piccolo riquadro delle seconde risposte (in alto a sinistra), una sintesi lessicale motivazionale (LEX-M, in CS-1) e una sintesi comparativa (CS-2) fra le denominazioni per la «baratte droite» (la zangola verticale) e per il «babeurre». Nella prima sintesi, la classificazione motivazionale va di pari passo con l'indagine etimologica sulle denominazioni per il «latticello di burro», in parte trasparenti (con il riutilizzo dell'iconimo \{beurre\}, spesso nella forma sintagmatica liquide/lait de beurre: 
triangolo/blu), in parte opachi e rappresentati prevalentemente dal tipo lessicale * bezèya. L'interpretazione etimologica di questa denominazione muove dalle risposte trasparenti di EM-Émarèse e AR-Arnad, dove l'iconimo di riferimento non è \{burro\} ma \{baratte\}/\{zangola\}, all'interno di un sintagma latte di zangola in cui il tipo lessicale utilizzato è *beus, denominazione attestata uniformemente per la Bassa Valle in APV 1-52 la baratte droite/la zangola verticale, dedicata a questo oggetto. Questa premessa permette non solo di interpretare il tipo *bezèya come una formazione aggettivale su *beus «zangola» (nel patois -èya è infatti il suffisso del participio passato femminile), ma anche di individuare una seconda famiglia motivazionale (quadrato/arancio) basata sull'adozione dell'iconimo \{baratte\}, che include il tipo *bezèya (arancio scuro), le denominazioni sintagmatiche del tipo «latte di zangola» (giallo) e anche le attestazioni di LS-La Salle e LT-La Thuile (arancio intermedio), dove il tipo *berayòn, più che direttamente da beurro «burro», sarà da intendere come un derivato di berée ( ${ }^{*}$ burraria), nome generale della zangola in queste località.

Questa configurazione permette di evidenziare l'uniformità motivazionale (anche se non lessicale-etimologica) di gran parte del territorio della Valle, da cui restano escluse solo le valli del margine meridionale più discoste dalle vie di transito principali; un'area uniforme in cui il «latticello di burro» e la «zangola» risultano fra loro lessicalmente collegati. Al quadro storico-linguistico di questo piccolo settore lessicale la sintesi comparativa in CS-2 aggiunge un altro tassello, relativo al rapporto diacronico fra $\mathrm{i}$ derivati della due basi *beus e * burraria $^{6}$ che si ritrovano tanto nelle denominazioni della «zangola verticale» che in quelle del «latticello di burro»: la distribuzione di quadrato/arancio e stella/bruno nella cartina mostra con evidenza una dinamica di opposizione est-ovest, con un'area orientale in cui la prima base vale per entrambi i referenti, una occidentale in cui si attesta la presenza di *burraria, una centrale in cui *beus resiste per la denominazione ormai opaca del «latticello» ma è sostituito da *burraria (che si connota così come innovazione) per il nome dello strumento. 
Figura 6. - Sintesi lessicali «motivazionali» e «comparative, da APV 1-62 le babeurre.

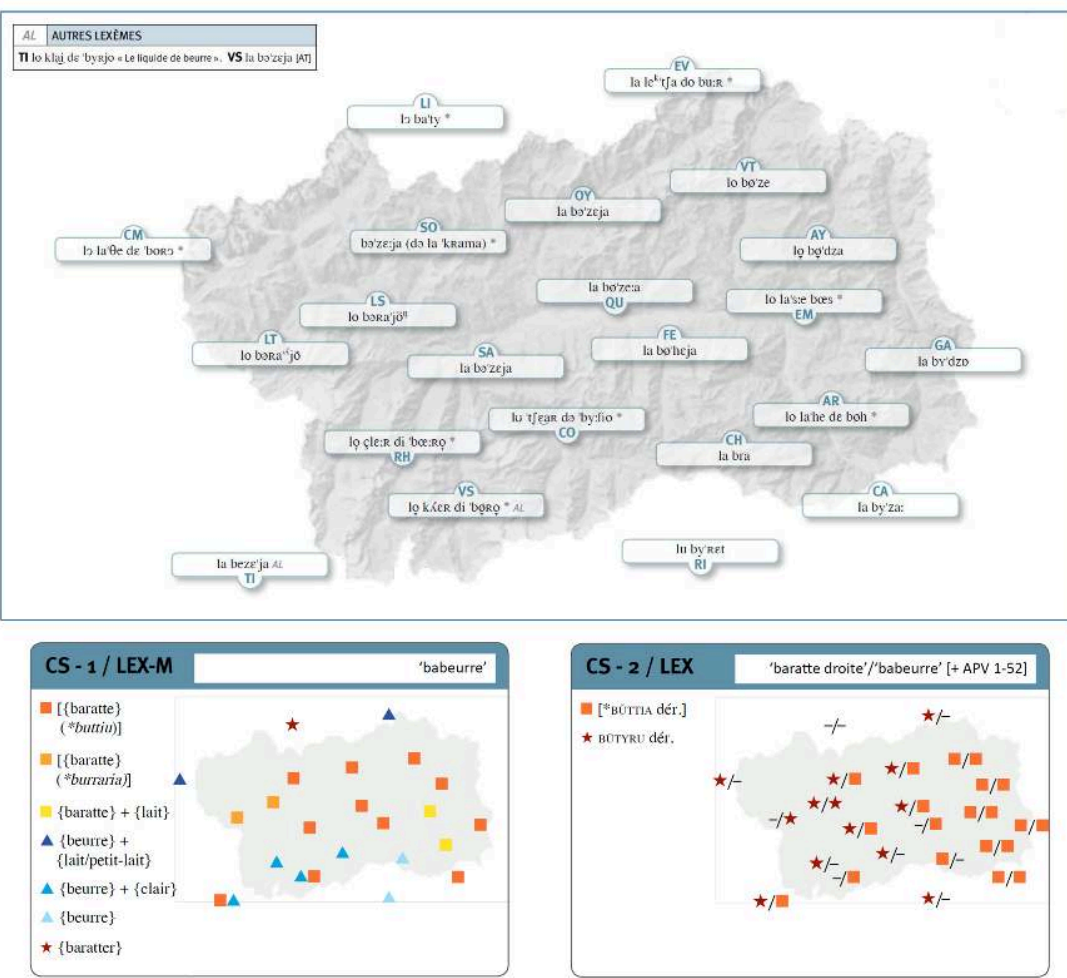

\subsection{Classificazione linguistica: la fonetica}

Le carte simboliche di tipo fonetico dell'APV descrivono la distribuzione di un singolo fenomeno fonetico evolutivo così come esso si presenta in un lessema (o in una serie di lessemi, laddove il fenomeno vi ricorra) attestato nella voce dove la carta compare e, a volte, anche in altre voci dell'atlante, che in questo caso vengono menzionate fra parentesi quadre (v. fig. 7, B.). Il fonema etimologico oggetto di analisi, accompagnato spesso dall'indicazione del suo contesto fonetico specifico, viene descritto sinteticamente nell'intestazione della legenda e fatto seguire dalla base etimologica del lessema esaminato, in cui il grafema in grassetto ribadisce per maggior chiarezza posizione, natura fonica (in particolare, la «quantità» breve o lunga per le vocali toniche del latino), e contesto di occorrenza del fonema ${ }^{7}$. 
Figura 7. - Esempi di sintesi fonetiche (v. 3.3).
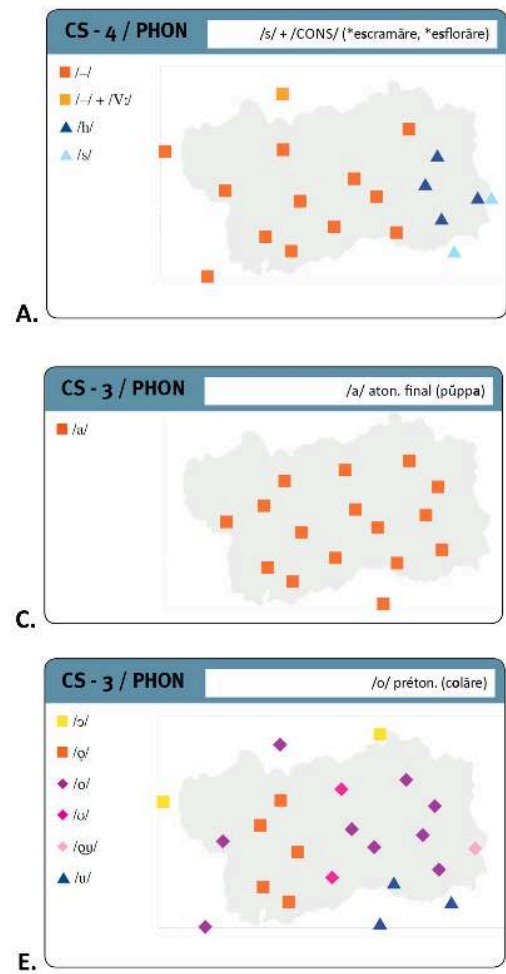
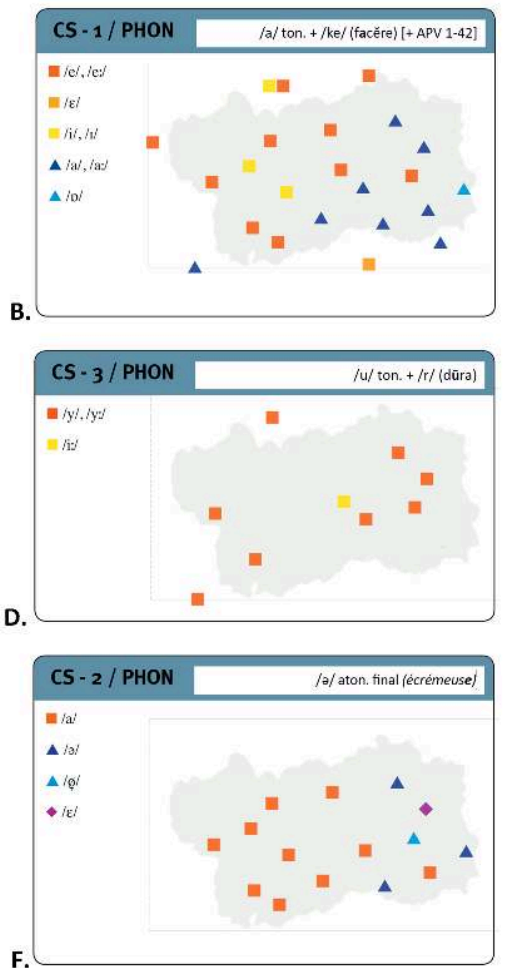

19 Come si intuisce dal loro numero elevato (229), le sintesi fonetiche sono state realizzate con larghezza e tutte le volte che, vuoi la distribuzione geolinguistica, vuoi la natura qualitativa dei fenomeni di evoluzione fonetica, sono state ritenute meritevoli di attenzione e la quantità dei dati disponibili (numero di punti in cui compare il lessema) lo permetteva. Pur prevalendo ovviamente i casi in cui si evidenziano situazioni di significativa opposizione fra aree (fig. 7, esempi A. e B.) ${ }^{8}$, non sono stati infatti tralasciati quelli in cui la distribuzione presenta caratteri di uniformità (fig. 7, esempi C. e D.), soprattutto quando i fenomeni osservabili risultassero particolarmente rilevanti nell'ambito dei caratteri fonologici tipici del francoprovenzale ${ }^{9}$. Nella scelta dei fenomeni, inoltre, si è cercato di dare rilievo anche a quelli che generalmente, nella tradizione degli studi di fonetica storica, sono ritenuti «secondari», come ad esempio l'ambito del vocalismo atono. Negli esempi E. (esito di /o/ pretonico in colāre) e F. (trattamento della /ə/ atona finale nel prestito dal francese écrémeuse ${ }^{10}$ si osserva ad esempio la consueta polarizzazione est-ovest delle soluzioni, significativa soprattutto nel secondo caso, dove l'Alta Valle mostra predilezione per l'integrazione del prestito nel sistema fonetico dialettale (restituzione della /a/ atona finale), la parte più a contatto con l'area italo-romanza, invece, per l'adozione come forestierismo (mantenimento dell'indistinta francese o adattamento superficiale in $/ ø / o / \varepsilon /$ ).

Sul piano metodologico, sarà poi opportuno far notare come soprattutto a livello di fonetica il processo di sintesi debba fare i conti con un aspetto connaturato alle operazioni di classificazione, che risiede nel margine di "arbitrarietà» lasciato al classificatore dalla complessità dei fenomeni osservati, poiché (come si diceva) ogni classificazione è già «interpretazione del dato». 
Figura 8. - Altri esempi di sintesi fonetiche (v. 3.3).
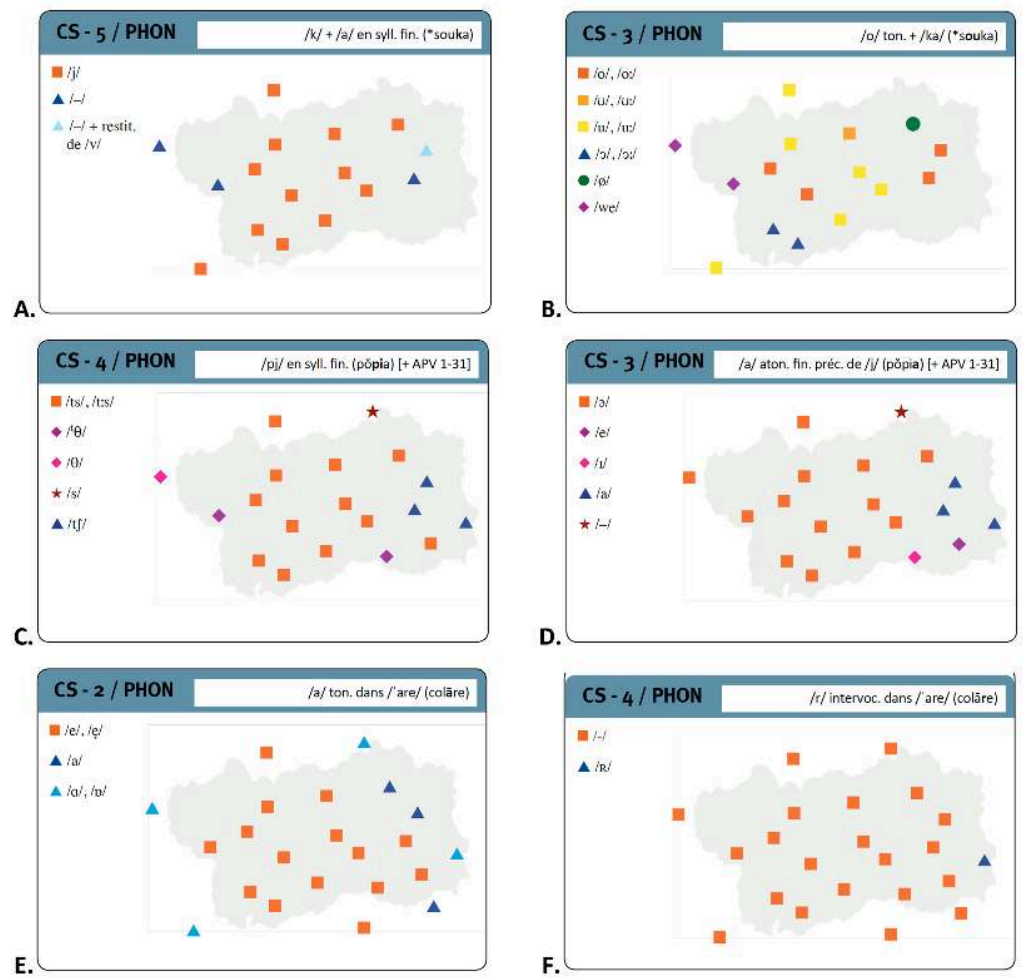

21 Nelle sintesi riportate in fig. 8 vengono proposti alcuni esempi significativi dei criteri adottati per questa operazione. Si noterà allora che, se nell'esempio A. (tratto da APV 1-13 le lait produit par une traite) la simbolizzazione dei risultati si appoggia su una classificazione piuttosto incontrovertibile degli esiti derivanti da / $\mathrm{k} /$ etimologica seguita da /a/ in posizione atona finale ${ }^{11}$, in $\mathrm{B}$. l'attribuzione ad una stessa classe simbolica (quadrato) degli esiti compresi fra il grado medio-alto /o/ e alto /u/ della /o/ tonica ricostruita per la base etimologica *souka, ad un'altra classe invece (triangolo) di quello medio aperto / $\mathrm{J} /$, rappresenta indubbiamente una scelta del classificatore ${ }^{12}$; e analogamente «arbitraria», nell'esempio C. (da APV 1.32 l'écumoire, con dati aggiuntivi da APV 1-31 l'écrémoire), è l'articolazione in quattro classi distinte degli esiti del gruppo /pj/ nella base tardo-latina pŏpia 'mestolo' (FEW IX, 176), laddove la considerazione ad esempio del solo «modo» di articolazione ne avrebbe potuto individuare due («affricate», comprendente gli esiti /ts/ e / $\mathrm{t} /$; «fricative», per / $\theta / \mathrm{e} / \mathrm{s} /$ ), con un grado intermedio $/ \theta /$ di mantenimento residuale dell'elemento occlusivo.

A questo problema metodologico si è cercato di ovviare essenzialmente attraverso l'adozione di un principio di estrema «analiticità» nell'osservazione dei fenomeni fonetici, procedendo preferenzialmente a sintesi separate per i singoli foni etimologici anche laddove essi compaiano in contesti potenzialmente analizzabili congiuntamente. La sintesi in fig. 8/D., sempre basata sull'ultima sillaba della base etimologica pŏpia, mostra un caso in cui l'articolazione analitica è quasi doverosa, data l'importanza tipologica (come detto sopra in nota 11) del trattamento palatale o no di /a/ atona finale; al di là di questa considerazione, la moltiplicazione dei punti d'osservazione fonetici ottenuta attraverso questa articolazione ottiene peraltro anche l'effetto di ridurre statisticamente l'impatto delle singole scelte dell'osservatore-classificatore. 
Con questo fine, il principio analitico è stato applicato in modo piuttosto sistematico nelle sintesi fonetiche, come mostrano gli esempi in figg.8/E. e F., dove sintesi differenziate sono state applicate, nel contesto del morfema -āre dell'infinito del verbo colāre (APV 1-20; v. nota 12), agli esiti rispettivamente di /a/ tonico e di /r/ intervocalico e dove i risultati quantitativi del metodo possono essere misurati sul parametro della "convergenza», che oscillerà all'interno di un gradatum che va dalla convergenza assoluta a quella nulla.

Per completezza di illustrazione, la fig. 9 mostra un altro esempio di questa delicata metodologia, corredata della carta analitica di partenza e applicata questa volta agli esiti del verbo latino fŭndĕre sotto il triplice aspetto dell'esito timbrico di /o/ tonico in contesto nasale (CS-2), del risultato della nasale preconsonantica stessa (CS-3), infine di quello dell'esito delle due sillabe atone finali, caso in cui contrariamente al solito si è scelta una classificazione di tipo «olistico» giustificata sul piano dell'analisi della struttura sillabica delle parole etimologicamente proparossitone (CS-4).

Figura 9. - Una carta analitica (da APV 1-68 faire fondre le beurre) e le sintesi fonetiche derivate.

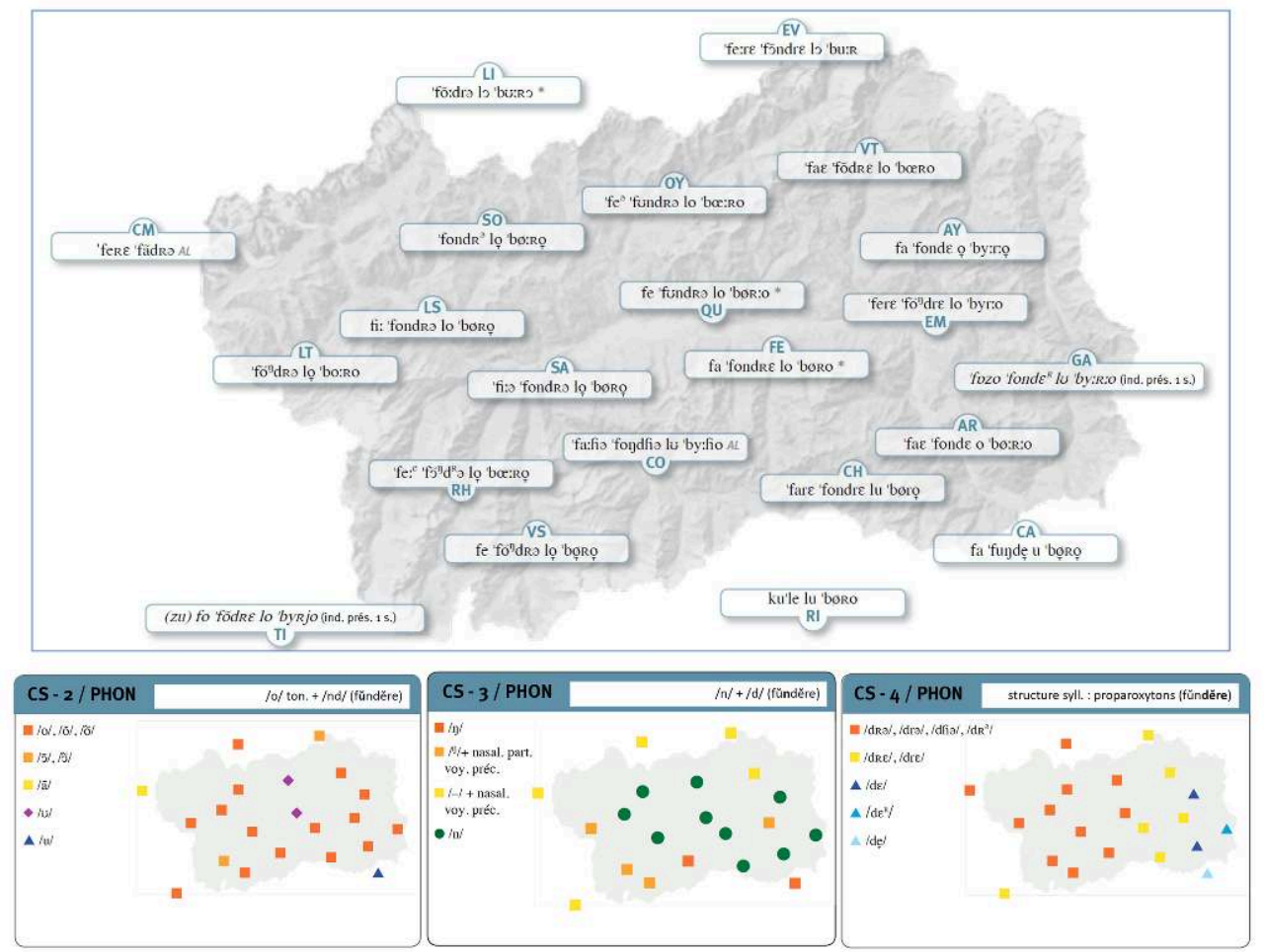

\subsection{Rappresentatività del campione e configurazioni geolinguistiche emergenti}

Abbiamo mostrato come l'approccio metodologico seguito dall'APV nella redazione delle sintesi linguistiche sia stato finalizzato a conseguire risultati importanti in termini di «rappresentatività» del campione, operando sul piano quantitativo della «numerosità» e contemporaneamente cercando di limitare l'impatto della arbitrarietà insita nelle scelte classificatorie con il ricorso piuttosto sistematico ad un approccio estremamente analitico ai fenomeni linguistici indagabili. Questo approccio ci pare 
costituisca la premessa ermeneutica necessaria a qualsiasi pretesa di successivo utilizzo dei dati APV nella chiave «dialettometrica» che proporremo nella parte conclusiva (4).

Inoltre, è vero anche che il confronto continuo con le configurazioni geolinguistiche emergenti dalle sintesi simboliche ha determinato da un lato una particolare attenzione nel ricercatore rispetto alla regolarità di alcune di esse, dall'altro ha fatto anche emergere progressivamente il carattere non isolato di altre, meno attese e per questo più interessanti.

Se infatti gli studi linguistici sul francoprovenzale valdostano hanno da tempo consolidato uno scenario geolinguistico secondo il quale la regione risulta divisa «in due aree linguistiche, l'alta e la bassa Valle [...]. La prima [...] ha risentito dell'influenza dei patois savoiardi e del Vallese [...]. La bassa Valle è, da una parte, l'area più conservatrice [...], dall'altra [...], ha subito l'influenza del Piemontese» (Favre, 2002: $141)^{13}$, la casistica ampia offerta dalle carte sintetiche dell'APV ha portato alla luce alcune novità, che mostrano un panorama sicuramente non così «dicotomico» come quello proposto sopra. Ciò che si è infatti potuto osservare è, da un lato, che la variabilità interna spesso osservata (ad esempio, sul piano fonetico, da Keller, 1958; e nuovamente da Favre, 2002) non riguarda nella stessa misura tutto il territorio valdostano, ma tende piuttosto a crescere man mano che ci si sposta verso oriente, e che anzi l'Alta Valle, nel suo complesso, mostri fenomeni significativi di convergenza; dall'altro, che pare discutibile l'assunto secondo il quale la stessa Alta Valle riveli complessivamente un orientamento verso il retroterra francoprovenzale d'oltralpe e forse anche che, in direzione opposta, la Bassa Valle risenta di un'influenza diretta del piemontes $\mathrm{e}^{14}$. Nella parte che segue ci proponiamo di sottoporre queste ipotesi critiche al banco di prova di un confronto impostato su base quantitativa e volto nel complesso a disegnare il quadro che sembra emergere da un campione significativo della cartografia sintetica contenuta nell'imminente primo volume dell'APV.

\section{Dall'interpretazione del dato alla «distanza linguistica»: approcci dialettometrici}

28 Oltre che operazione funzionale all'interpretazione immediata dei dati forniti da un atlante, la redazione di carte sintetiche rappresenta, come abbiamo mostrato, il passo indispensabile in vista di prospettive di studio successive e di portata più ampia, volte alla delineazione complessiva della caratterizzazione geolinguistica dell'area in esame e, in particolare, alla valutazione del fattore della «variazione linguistica interna» di un campione geolinguistico, definibile in termini di distanza linguistica reciproca fra gli elementi del campione stesso (i punti d'inchiesta dell'atlante).

Sarà naturalmente anche opportuno premettere che rispetto ai dati APV ci si muove comunque nel quadro di varietà linguistiche affini, caratterizzate nel complesso da una bassa distanza strutturale reciproca; e che, conseguentemente, l'obiettivo soggiacente alle operazioni di misurazione della distanza linguistica sarà tendenzialmente quello di «amplificare» e rendere più visibili le differenze esistenti, anche laddove esse (sul piano più generale della tipologia linguistica) non siano in realtà particolarmente impattanti. Nel caso specifico, l'approccio è tuttavia giustificato dal fatto di muoversi nella scala dimensionale di un atlante regionale, oltretutto riferito ad un territorio particolarmente ridotto $\left(3261 \mathrm{~km}^{2}\right)$. 


\subsection{Fondamenti teorici}

In dialettologia, l'approccio maggiormente sistematico alla misurazione della distanza linguistica è costituito indubbiamente dall'approccio dialettometrico, inaugurato negli anni Settanta da Jean Séguy (1973) e poi elaborato compiutamente dalla cosiddetta «scuola salisburghese» a partire dagli anni Ottanta del secolo scorso (Goebl, 1984 e ora, per una sintesi aggiornata, Goebl, 2016), uno dei cui strumenti principali di indagine è rappresentato dalle cosiddette "Carte di Similarità» (CSim).

Una Carta di Similarità, in sostanza, elabora in forma grafica degli indici numerici. Questi sono rappresentati dagli Indici di Similarità, ovvero i valori percentuali di corrispondenza fra i punti di una rilevazione geolinguistica e un punto particolare scelto come termine di riferimento, rispetto a un numero statisticamente significativo di realizzazioni linguistiche osservabili comparativamente per mezzo di carte di atlante (Goebl, 2016: 563-567) e classificabili come «corrispondenti» o «non corrispondenti» (operazione detta Tassazione). La somma delle classificazioni determina un'oscillazione che va dal $100 \%$ di similarità attribuibile teoricamente al punto di riferimento stesso e l'altrettanto teorico $0 \%$ di un punto che diverga costantemente da esso.

L'elaborazione nella forma grafica tradizionale della Dialettometria di scuola salisburghese prevede la realizzazione di carte (dette "coropletiche») in cui ciascun punto d'atlante è rappresentato da un poligono la cui colorazione suggerisce la complessiva "prossimità» (colori caldi della scala cromatica) o «distanza» linguistica (colori freddi) rispetto al punto di riferimento scelto (rappresentato da un poligono bianco). Il punto di riferimento può essere costituito da una varietà dialettale «centrale» o "periferica» rispetto all'area d'osservazione, oppure anche da un tipo linguistico non geolocalizzato, come sarebbero l'italiano o il francese standard per un'indagine sui dialetti rispettivamente italo- e galloromanzi (Goebl, 2016: 567-568).

3 Goebl (e con lui Roland Bauer) hanno esercitato in svariati studi la metodologia dialettometrica, applicandola soprattutto ai materiali dell'AIS, dell'ALF e a quelli del loro ALD, e quindi in particolare al ladino dolomitico e alle aree circonvicine ${ }^{15}$. Riprendendo i principi-cardine dell'approccio, cercheremo ora di applicarli al contesto particolare rappresentato dal nostro atlante regionale.

\subsection{Misurazione della distanza linguistica media rispetto al patois «centrale»}

Sulla base delle ipotesi caratterizzanti delineate sopra (3.4), si è scelta come punto di riferimento per le misurazioni una varietà dialettale definibile come "centrale», che è stata identificata nel punto di SA-Sarre, comune situato nella Valle Centrale al confine con il capoluogo Aosta.

\subsubsection{Metodo di rappresentazione}

L'indagine dialettometrica si è basata su un campione costituito dalle carte sintetiche linguistiche (lessicali, morfosintattiche e fonetiche) contenute nelle prime 50 voci del volume-pilota Le lait et les activités laitières, per un totale di 136 sintesi (CS-LEX: 46; CSMORPH: 6; CS-PHON: 84) ${ }^{16}$. 

la conseguente attribuzione dei simboli, considerati secondo le classi $(\mathrm{F}=$ «forma») e le sottoclassi $(\mathrm{C}=$ «sfumature di colore») simboliche adottate, sono state assunte come base per la tassazione e per la valorizzazione numerica della similarità (VAL) con SASarre di ogni punto per ognuna delle 136 sintesi, procedendo secondo l'algoritmo generale seguente: $\mathrm{VAL}=1$ per $\mathrm{F}$ e $\mathrm{C}$ uguali a SA-Sarre; $\mathrm{VAL}=0,75$ per $\mathrm{F}$ uguale a SA-Sarre ma $\mathrm{C}$ diverso; $\mathrm{VAL}=0$ per $\mathrm{F}$ e $\mathrm{C}$ diversi da SA-Sarre. $\mathrm{E}$ stato inoltre introdotto un $\mathrm{VAL}=0,5$ per i casi in cui l'utilizzo per un punto d'inchiesta della classe simbolica «rombo/viola» volesse rappresentare una classe intermedia fra quella attribuita a SA-Sarre (generalmente «quadrato/arancio») e quella posta in opposizione (generalmente «triangolo/blu»).

L'attribuzione dei punteggi ha determinato così una Matrice di Similarità, in cui ciascun punto d'inchiesta esibisce un punteggio (ipoteticamente compreso fra 0 e 136), che, riportato in base 100, determina il valore che definiamo Percentuale di Similarità (\% IM) con il punto di riferimento centrale. Nelle rappresentazioni cartografiche che seguono, il valore percentuale conseguito da ogni punto d'inchiesta è stato convertito in un gradiente cromatico che va dal rosso $\left(\% \mathrm{SIM}_{\mathrm{IM}}=100\right)$ al verde limone $\left(\% \mathrm{SIM}_{\mathrm{IM}}=0\right)$ e che è stato attribuito ad un cerchio trasparente sovrapposto al punto d'inchiesta, il cui colore rappresenta pertanto la distanza linguistica di quel punto dal patois di Sarre ${ }^{17}$.

\subsubsection{Similarità linguistica generale}

Figura 10. - Carte di Similarità rispetto al punto di riferimento SA-Sarre (patois «centrale»). CSim1: similarità linguistica media.

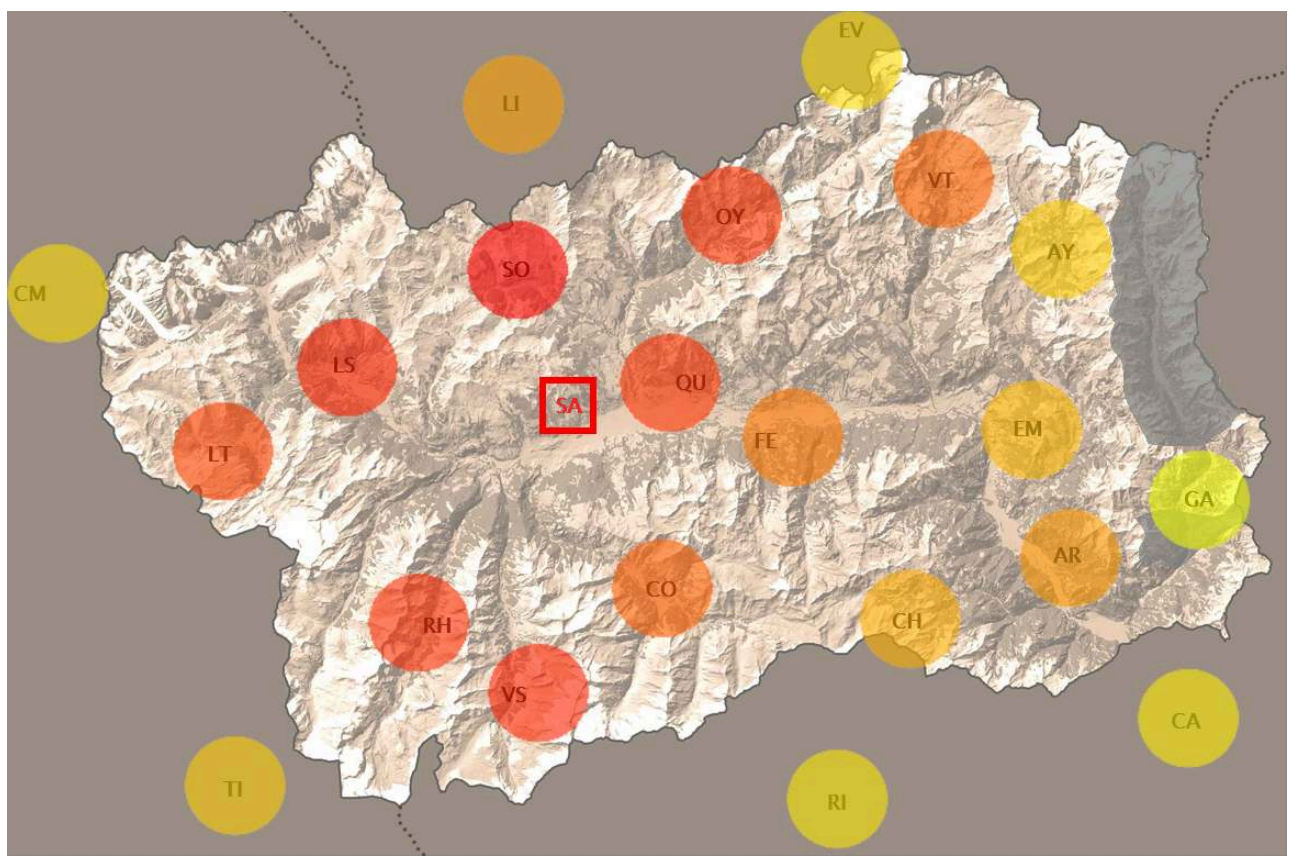

Come si può vedere nella prima delle nostre elaborazioni (fig. 10, che rappresenta appunto la similarità linguistica generale delle diverse varietà dialettali con il patois centrale), intorno al punto di riferimento di SA-Sarre si disegna un'area di relativa uniformità convergente decisamente orientata verso l'Alta Valle, che vede il punto SOSaint-Oyen (valle del Gran San Bernardo) registrare la \%SIM maggiore (94,1). Gli altri 
punti dell'Alta Valle (compreso il punto OY-Oyace, nella Valpelline che converge geograficamente verso la conca di Aosta) costituiscono un insieme con valori superiori a \%SIM 75, i cui limiti inferiori sono rappresentati da QU-Quart (\% SIM 80,2), sul fondo valle ad est del capoluogo, e LT-La Thuile (\%SIM 76,9), nella valle del Piccolo San Bernardo.

Spostandosi verso est, i punti di VT-Valtournenche (valle del Cervino, che sbocca nel Fondo Valle allo snodo della conca di Châtillon, dove la Dora Baltea varia la direzione del suo corso da ovest-est a nord-sud), FE-Fénis (valle centrale, a $15 \mathrm{~km}$ da Aosta) e COCogne (valle che si estende dal Gran Paradiso verso ovest e sbocca ad ovest di Aosta) mostrano una sorta di area obliqua intermedia (\%SIM comprese fra CO 70,3 e FE 64,2), corrispondente al passaggio delle maggiori isoglosse tradizionalmente individuate (cfr. ad es. Perron, 1995). Si noterà in proposito che infatti, nel passaggio fra i due gruppi Alta Valle/Area Intermedia, si registra lo stacco maggiore delle \%SIM del campione (-6,6 fra LT e CO).

La Bassa Valle registra sempre \%SIM inferiori a 60, con la punta di AR-Arnad (56,1), più lontana da SA-Sarre ma situata sul fondo valle. I punti di AY-Ayas (nell'omonima valle che sbocca a metà del tratto nord-sud della valle della Dora), EM-Émarèse (sull'altopiano che affaccia sul limite nord dello stesso tratto) e CH-Champorcher (unico punto del tratto posto sul versante destro del fiume, nell'omonima valle laterale) registrano valori compresi fra 50,7 (CH) e 42,7 (AY), mentre un discorso a parte merita GA-Gaby. Questo punto di inchiesta (situato nella media Valle del Lys che sbocca al confine col Piemonte e isolato fra i comuni walser di Gressoney-La Trinité a nord, Issime a sud) riporta infatti $\mathrm{i}$ valori di similarità in assoluto più bassi del campione $(28,1)$.

41 Fra i punti esterni (tutti comunque più vicini linguisticamente a SA-Sarre di GA-Gaby), la distanza linguistica maggiore si registra nei punti piemontesi (CA-Carema \%SIM 35,5; RI-Ribordone \%SIM 37,0), mentre sarà da notare il valore relativamente alto $(54,1)$ del punto vallesano di LI-Liddes, località posta subito al di là del Gran San Bernardo, che si avvicina ai valori più alti della Bassa Valle valdostana e distacca di 11,4 punti il secondo punto d'Oltralpe per similarità (TI, al di là del Piccolo San Bernardo: \%SIM 42,9).

42 La rappresentazione dialettometrica pare innanzitutto confermare l'ipotesi di una convergenza significativa dei patois dell'Alta Valle, individuando però meglio i limiti $\mathrm{e}$ le direttrici geografiche di questa dinamica convergente, nella quale lo spostamento verso est nella valle centrale, e, in direzione opposta, verso l'area del Piccolo San Bernardo che conduce verso la Tarentaise (Savoia), determinano l'incontro di varietà in parte divergenti da SA-Sarre, come QU-Quart (località molto vicina al centro del sistema che esibisce però un'incipiente divergenza linguistica) e LT-La Thuile; cosa che non avviene invece sull'asse che conduce al Gran San Bernardo in direzione del Vallese francofono svizzero. Essa dimostra poi l'esistenza di un'opposizione Alta Valle/ Bassa Valle, individuando però anche più chiaramente un'Area Intermedia di transizione, orientata su un'asse obliquo nord-est/sud-ovest; ed evidenzia infine la posizione particolare di Gaby, che si configura (e per distacco) come il patois più distante da quello «centrale» preso come riferimento, inclusi i punti di controllo non valdostani. 


\subsubsection{Variabili strutturali}

I risultati del quadro generale delineato dalla sintesi dialettometrica generale hanno suggerito di sottomettere i dati APV ad ulteriori indagini quantitative, basate sulle seguenti domande di ricerca: cosa accade se ritagliamo all'interno del corpus dei sottoinsiemi statistici (Subsets) sulla base di variabili indipendenti e significative? Quanto variano, in questo caso, i valori di similarità linguistica e quali località sono sottoposte a una maggiore variazione? cioè alla differenza che può esistere fra la convergenza in ambito da un lato lessicale, dall'altro fonetico di due sistemi linguistici. Una visualizzazione di questa variabile si può ottenere facilmente processando separatamente, con lo stesso sistema adottato in precedenza, i due Subsets relativi alla variabile in oggetto, e cioè le 46 CS-LEX e le 84 CS-PHON (fig. 11).

Figura 11. - Carte di Similarità rispetto al punto di riferimento SA-Sarre (patois «centrale»). Variabili linguistiche strutturali (lessico e fonetica).

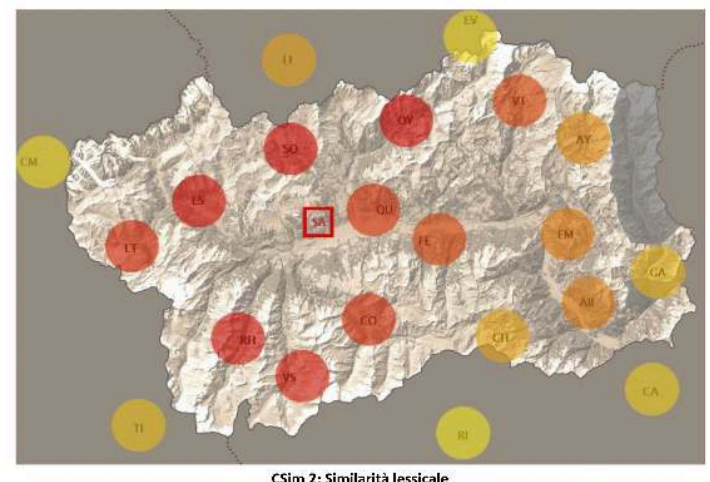

CSim 2: Similarità lessicale

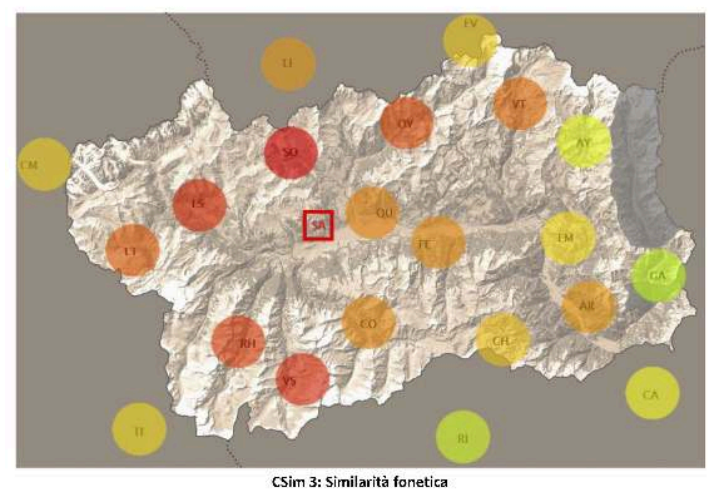

In linea generale, nel confronto visivo fra le due sintesi emerge immediatamente un generale aumento di convergenza (prevalenza di colori «caldi») per il lessico e una speculare diminuzione (prevalenza di colori «freddi») per la fonetica. Il risultato è in parte atteso e anche statisticamente condizionato sia dalla diversa numerosità dei campioni, sia soprattutto dalla natura qualitativa diversa delle analisi lessicale e fonologica, laddove nella seconda si tende (come si sarà potuto apprezzare adeguatamente in 3.3) ad evidenziare maggiormente differenze anche minime di realizzazione linguistica. Ci concentreremo quindi essenzialmente sul rilevamento delle più evidenti variazioni di posizionamento dei punti d'inchiesta nel Ranking ottenuto 
nei due Subset lessicale e fonetico, rispetto al Ranking precedente, indicative della natura della convergenza/divergenza linguistica rispetto al patois centrale.

Il quadro che emerge dal confronto è piuttosto chiaro e anche, a nostro avviso, significativo. Sul piano lessicale, si osserva un marcato «compattamento» dell'area regionale valdostana, dato che i 5 valori minori di similarità sono registrati stavolta dai punti esterni RI-Ribordone (33,8), CM-Les Contamines-Montjoie (36,5), EV-Évolène $(36,7)$, CA-Carema (39) e TI-Tignes $(43,6)$, cui seguono i punti valdostani GA-Gaby $(44,7)$ e $\mathrm{CH}$-Champorcher $(53,1)$; osservazione che dimostrerebbe una generale «convergenza culturale» dell'area, dato che il livello lessicale è quello naturalmente più sottoposto a fenomeni di circolazione che tendono diacronicamente a modificarlo. È invece sul piano della fonetica (collegato a una dimensione più strutturale e «fondamentale» del linguaggio) che la divergenza già riscontrata per l'area della Bassa Valle si manifesta più evidentemente e con una precisa marcatura geolinguistica: sono infatti i tre punti in altura situati sul versante sinistro della Dora (EM-Émarèse, \%SIM 42,0; AY-Ayas \%SIM 26,3 ; GA-Gaby \%SIM 16,4) a registrare gli spostamenti più marcati verso il fondo della graduatoria di similarità fonetica (ranking -6 per AY e EM; -5 per GA), evidenziando una divergenza linguisticamente più «profonda» di questa sub-area valdostana in rapporto a quella occidentale. Una configurazione opposta emerge invece per i punti francoprovenzali transalpini di EV-Évolène (\%SIM 45,5) e CM-Les Contamines-Montjoie (\%SIM 44,4), meno simili sul piano lessicale ma maggiormente convergenti su quello fonetico (ranking +5 in entrambi i casi).

\subsubsection{Variabili diatopiche «bifocali»}

Come detto sopra (4.1), nelle applicazioni dialettometriche la variabilità connessa alla dimensione diatopica viene generalmente sondata spostando semplicemente il punto di riferimento in una zona periferica dell'area linguistica indagata. Scegliamo invece qui un approccio (che ci risulta non avere precedenti), basato sul rilevamento di un altro tipo di configurazioni diatopiche (che chiamiamo «bifocali») suggerite dalle rilevazioni precedenti e definibili come «assi direzionali» che dal centro della regione muovono in direzioni opposte: verso la Bassa Valle (e quindi verso il confine col Piemonte) oppure verso il retroterra francoprovenzale transalpino.

Per sondare questa dimensione in termini dialettometrici, si è proceduto ad estrarre dal corpus originale ulteriori Subsets costruiti sulla base del confronto fra i dati relativi al solito punto di SA-Sarre e quelli di due località attestanti gradi «medi» di similarità (per garantire un campione sufficientemente ampio) e poste su questi assi direzionali, in particolare EM Émarèse per il primo scenario (\%SIM media 48,4) e LI-Liddes per il secondo (\% IIM 54,1). In entrambi i casi, il confronto è stato istituito sulla base sia della convergenza dei dati ( $\mathrm{SA}=\mathrm{EM}$ e SA $=\mathrm{LI}$; punteggio 1 ), anche parziale $(\mathrm{SA} \approx \mathrm{EM}$ e SA $\approx \mathrm{LI}$; punteggi $0,75 \circ 0,5)$, sia della loro divergenza $(\mathrm{SA} \neq \mathrm{EM}$ e $\mathrm{SA} \neq \mathrm{LI}$; punteggio 0 ), costruendo così quattro Subset «bifocali».

In fig. 12 si presentano le elaborazioni relative ai due Subset SA-EM (Asse Aosta-Bassa Valle), tratte rispettivamente da quello che elabora dati di coincidenza con EM-Émarèse (CSim4, in alto; 60 sintesi elaborate) oppure di divergenza (CSim5, in basso; 52 sintesi) rispetto al patois centrale. Come si può notare, la configurazione cromatica in CSim4, salvo un lieve aumento dei valori per gran parte delle località (in particolare per quelle che circondano EM, che ovviamente riporta una \%SIM uguale a 100) ${ }^{18}$, non presenta 
sostanziali differenze distributive rispetto a quella della similarità linguistica generale presente in fig. 11. Questo significa che, anche selezionando solo i fenomeni linguistici in cui centro e periferia orientale (Bassa Valle) tendono a convergere, il rapporto generale di similarità fra i punti indagati (compresi quelli transalpini) e il centro del sistema non cambia molto, poiché negli altri punti della Bassa Valle l'aumento atteso (che pure si produce e raggiunge il suo massimo con AY-Ayas, +20,9) non basta a produrre scavalcamenti di sorta, né rispetto ai punti dell'Alta Valle, e neppure rispetto al punto vallesano di LI-Liddes. Nello scenario di divergenza Alta Valle/Bassa Valle proposto invece da CSim5, quella che si disegna è una marcata frattura fra due aree: da un lato un'Alta Valle allargata alla fascia intermedia rappresentata dai punti VTValtournenche, FE-Fénis e CO-Cogne, dall'altro tutti gli altri punti, compresi (si noti bene) tutti quelli transalpini tranne in parte LI-Liddes. Una bipartizione che presenta due «salti» percentuali decisamente vistosi: di 14,1 punti fra LT-La Thuile (Alta Valle) e Co-Cogne (fascia intermedia) e di 17,9 fra Fénis (fascia intermedia) e AR-Arnad (Bassa Valle). Quando perciò Alta e Bassa Valle non coincidono, è più facile della media che il fenomeno linguistico in questione abbia una diffusione intra-regionale valdostana (e non estesa all'area transalpina) e limitata probabilmente all'area più occidentale della regione o, al limite, a quella intermedia: una prova abbastanza evidente del ruolo svolto da Aosta e dall'area centrale dei patois valdostani in direzione di una seppur parziale e geograficamente non estesissima uniformazione linguistica.

Figura 12. - Carte di Similarità rispetto al punto di riferimento SA-Sarre (patois «centrale»). Configurazione diatopica 2: asse Aosta-Bassa Valle.

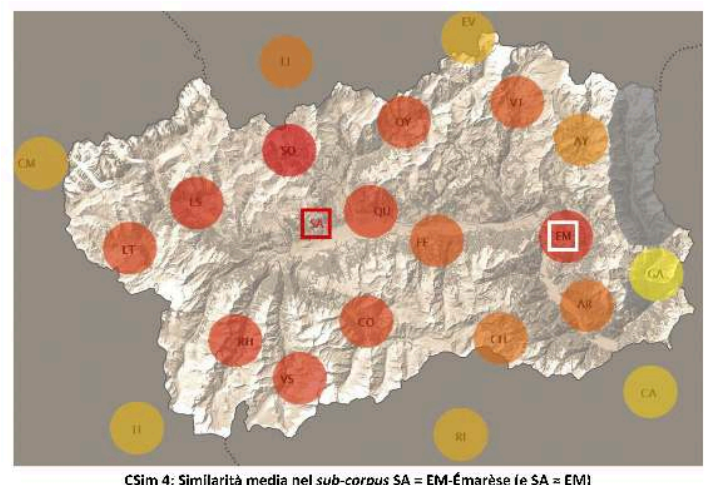

CSim 4: Similarità media nel sub-corpus SA = EM-Emarèse (e SA = EM)

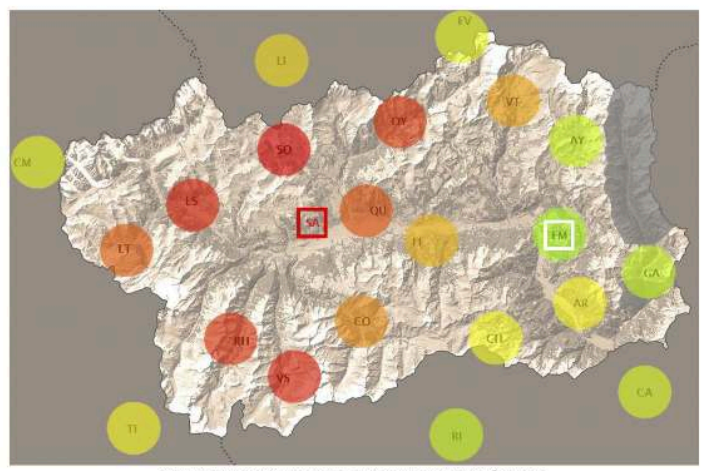

CSim 5: Similarità media nel sub-corpus SA ₹ EM-Émarèse

51 In fig. 13 si presentano le analoghe elaborazioni relative al Subset SA-LI (asse AostaRetroterra francoprovenzale transalpino), che si riferisce ad una località che nei dati APV si rivela spesso come il punto francoprovenzale esterno più convergente con il nostro baricentro linguistico regionale. 
il focus d'osservazione si sposti su questo asse, lo scenario di convergenza (CSim6, in alto; 58 sintesi) mostra alcune variazioni rispetto al corpus totale: pur nel quadro del medesimo aumento pressoché generale della convergenza segnalato sopra, piuttosto visibile però stavolta anche negli altri tre punti transalpini, è la situazione della Bassa Valle a presentare una configurazione particolare. Il mantenimento su valori medi dei punti più interni dell'area (quelli più vicini alla Valle Centrale: EMÉmarèse, $\mathrm{CH}$-Champorcher e AR-Arnad), e l'evidenziazione di una «corona» esterna con valori bassi, che include anche il punto di AY-Ayas (separato in questo caso dalla vicina EM-Émarèse da 19,7 punti, a fronte dei soli 5,7 di distanza rilevabili nel corpus totale), suggerisce una relazione diretta fra perifericità geografica rispetto all'asse viario che attraversa la Valle e perifericità linguistica. Nel caso specifico della convergenza linguistica Aosta-Gran San Bernardo, insomma, le innovazioni convergenti che accomunano il patois centrale e il suo retroterra francoprovenzale settentrionale sembrano trovare proprio nella viabilità centrale (romana, medievale e moderna) il loro mezzo di diffusione.

Nella sintesi di divergenza (CSim7, in basso; 40 sintesi), invece, l'aspetto più appariscente sta nella compatta discesa verso valori minimi di tutti i punti francesi e svizzeri: quando un fenomeno linguistico del patois centrale non è condiviso al di là del Gran San Bernardo, insomma, è difficile che lo sia con gli altri punti del retroterra francoprovenzale.

Figura 13. - Carte di Similarità rispetto al punto di riferimento SA-Sarre (patois "centrale»). Configurazione diatopica 1: asse Aosta-Retroterra francoprovenzale transalpino.

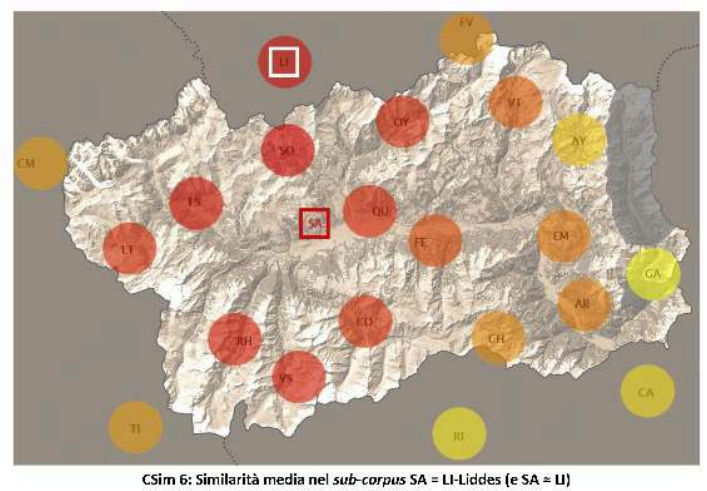

CSim 6: Similarità media nel sub-corpus SA = LI-Liddes (e SA $\approx$ U)

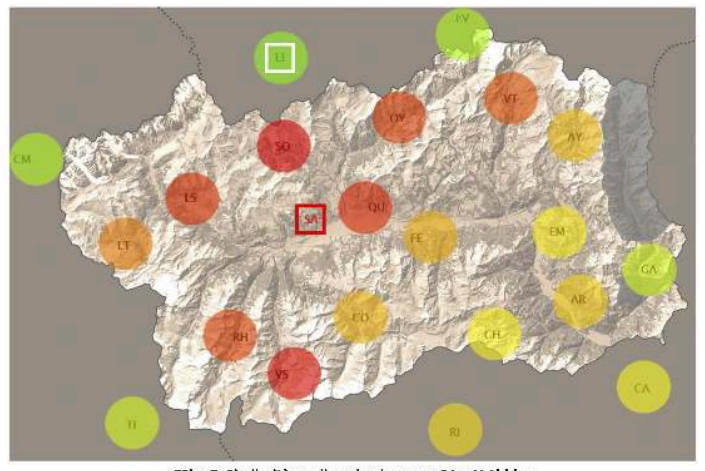

CSim 7: Similarità media nel sub-corpus 5A $\neq$ U-Liddes 


\section{Conclusioni}

Le sintesi dialettometriche proposte sembrano attestare, attraverso gli spunti di lettura geolinguistica che ne derivano, la produttività dello strumento adottato rispetto ai materiali APV, offrendo conferme significative di scenari geolinguistici consolidati, ma anche precisando meglio i limiti e i dettagli di questi e, soprattutto, rivelandone altri potenzialmente innovativi.

Pur nei limiti quantitativi di questo sondaggio, l'ipotesi scientifica dialettometrica appare insomma particolarmente promettente; soprattutto nella misura in cui si presta (attraverso la definizione di parametri differenziali di valutazione della similarità linguistica, rappresentati qui dai Subset «strutturali» e "diatopici» elaborati) ad indagare aspetti specifici delle dinamiche di evoluzione linguistica.

In presenza di una mole di dati maggiore (ottenibile anche solo attraverso la processazione totale delle sintesi linguistiche del primo volume APV), sarebbe ad esempio possibile sottomettere ad indagine aspetti qualitativi ancora più interessanti, che potrebbero prevedere il sondaggio separato di classi di lessemi distinti per strato etimologico (prelatino, latino, germanico) oppure la valutazione dell'impatto differenziato del vocalismo e del consonantismo (settori fonetici il cui comportamento differente è, come sappiamo, particolarmente rilevante nell'individuazione ascoliana $\mathrm{e}$ nella definizione stessa del francoprovenzale) o addirittura di aree ancora più ristrette della fenomenologia fonetica.

Per un'indagine dialettometrica approfondita sull'oggetto di studio rappresentato dal micro-territorio valdostano e dalla sua variabilità linguistica interna, spunti e materia prima su cui lavorare non paiono insomma mancare, al presente e (diremmo) per alcune generazioni di dialettologi.

\section{BIBLIOGRAFIA}

AIS = JABERG Karl \& JUD Jakob, 1928-1940, Sprach-und Sachatlas Italiens und der Südschweiz, Zofingen, Ringier.

ALD = GOEBL Hans \& BAUER Roland, 1998, Atlant linguistic dl ladin dolomitich y di dialec vejins I / Atlante linguistico del ladino dolomitico e dei dialetti limitrofi I / Sprachatlas des Dolomitenladinischen und angrenzender Dialekte I, Wiesbaden, Ludwig Reichert Verlag.

ALF = GILLIÉRON Jules \& EDMONT Edmond, 1902-1910, Atlas linguistique de la France, Paris, Champion.

AlineI Mario, 1994, L'Atlas Linguarum Europae: risultati, struttura, storia, prospettive, in P. Garcia

Mouton (a cura di), Geolinguistica. Trabajos europeos, Madrid, Consejo Superior de Investigaciones Cientificas, pp. 1-39.

AlineI Mario, 1997, Principi di teoria motivazionale (iconimia) e di lessicologia motivazionale

(iconomastica), in L. Mucciante e T. Telmon (a cura di), Lessicologia e lessicografia: Atti del XX Convegno della SIG (Chieti-Pescara, 12-14 ottobre 1995), Roma, Il Calamo, pp. 9-36. 
CUGNo Federica \& MASsoBRIo Lorenzo, 2010, Gli atlanti linguistici della Romània. Corso di geografia linguistica, Alessandria, Edizioni dell'Orso.

DAUZAT Albert, 1955, La méthode des Nouveaux Atlas linguistiques de la France, «Orbis», nº 1, pp. 22-31.

DEI = BATTISTI Carlo \& ALESSIO Giovanni (a cura di), 1950-1957, Dizionario etimologico italiano, Firenze, Barbèra.

DELI = CORTELAZzo Manlio \& Zolli Paolo, 1999, Dizionario etimologico della lingua italiana, Bologna, Zanichelli.

FAVRe Saverio, 2002, La Valle d'Aosta, in M. Cortelazzo, C. Marcato, N. De Blasi e G. P. Clivio (a cura di), I dialetti italiani. Storia, struttura, uso, Torino, UTET, pp. 139-150.

FEW = WARTBURG Walther VON (a cura di), 1922-1989, Französisches etymologisches Wörterbuch, Bonn et al., Zbinden et al.. Consultabile online su <https://apps.atilf.fr/lecteurFEW/>.

GOEBL Hans, 1984, Dialektometrische Studien. Anhand italoromanischer, rätoromanischer und galloromanischer Sprachmaterialien aus AIS und ALF, Tübingen, Niemeyer.

GOEBL Hans, 2003, Entwicklung des romanischen Sprachgrenzen: Italoromania und Ostalpenraum / Évolution des frontières des langues romanes : l'Italoromania et les Alpes orientales, in G. Ernst, M.-D. Glessgen, C. Schmitt et al. (a cura di), Romanische Sparachgeschichte / Histoire linguistique de la Romania, t. 1, pp. 634-645.

GoEBL Hans, 2016, La geografia linguistica, in S. Lubello, Manuale di linguistica italiana, Tübingen, Niemeyer, pp. 553-580 [Manuals of Romance Linguistics, vol. 13].

GRASSI Corrado, 1955-1957, Analisi delle caratteristiche lessicali della Valle d'Aosta in base ai materiali forniti dai tre Atlanti linguistici nazionali (ALF-AIS-ALI), «Romanistiche Jahrbuch», n 7 , pp. 55-65 e $\mathrm{n}^{\circ}$ 8, pp. 63-74.

KELLER Hans-Erich, 1958, Études linguistiques sur les parlers valdôtains, Bern, A. Francke.

MARTIN Jean-Baptiste, 1990, Francoprovençal, in G. Holtus, M. Metzeltin e C. Schmitt (a cura di), Lexicon der Romanistischen Linguistik, vol. V, 1, Tübingen, Max Niemeyer, pp. 671-685.

PERRON Marco, 1995, Unitarietà e variabilità lessicale nelle parlate francoprovenzali della Valle d'Aosta, in S. J. Woolf (a cura di), La Valle d'Aosta, Torino, Einaudi, pp. 205-218.

RAIMONDI Gianmario, 2016, Conflitti di lingue e di culture fra Gallo-Romània e Gallo-Italia. Una rilettura a partire dai dati lessicali dell'Atlas des patois valdôtains, in C. Calvo Rigual, L. Minervini e A. Thibault (a cura di), Actes du XXVII ${ }^{e}$ Congrès international de linguistique et de philologie romanes (Nancy, 15-20 luglio 2013). Consultabile online su <www.atilf.fr/cilpr2013/actes/section-11/CILPR-2013-11Raimondi.pdf> [ultimo accesso il 19 settembre 2019].

RAIMONDI Gianmario, 2017, Giving Their Voice Back to "Speech Communities": The APV, «Éducation et sociétés plurilingues», $\mathrm{n}^{\circ} 42$, pp. 9-18. Consultabile online su <http://journals.openedition.org/ esp/1071> [ultimo accesso il 12 settembre 2019].

RAIMONDI Gianmario, 2019, APV-Atlas des patois valdôtains, in T. Krefeld e R. Bauer (a cura di), Lo spazio comunicativo dell'Italia e delle varietà italiane. Korpus im Text. Consultabile online su $<$ www.kit.gwi.uni-muenchen.de/?p=12756> [ultimo accesso il 29/09/2019].

REP = CORNAGLIOTTI Anna (a cura di), 2015, Repertorio etimologico piemontese-REP, Torino, Centro Studi Piemontesi/Ca' dë Studi Piemontèis. 
SÉGUY Jean, 1973, La dialectométrie dans l'Atlas linguistique de la Gascogne, «Revue de linguistique romane», $\mathrm{n}^{\circ} 37, \mathrm{pp} .1-24$.

TUAILLON Gaston, 1972-1973, Questionnaire pour enquêtes dialectales en pays alpin, Grenoble, ELLUG.

TUAILLON Gaston, 2007, Le francoprovençal, t. 1, Aoste, Musumeci.

VINCENT Nigel, 1988, Latin, in M. Harris e N. Vincent (a cura di), The Romance Languages, New York, Oxford University Press, pp. 26-78.

\section{NOTE}

1. Punti interni (ovest-est/nord-sud): LT-La Thuile, LS-La Salle, RH-Rhêmes-Saint-Georges, VSValsavarenche, SO-Saint-Oyen, SA-Sarre, CO-Cogne, QU-Quart. OY-Oyace, FE-Fénis, CHChamporcher, VT-Valtournenche, AY-Ayas, EM-Émarèse, GA-Gaby, AR-Arnad. Punti esterni: LILiddes e EV-Évolène (Vallese svizzero), CM-Les Contamines-Montjoie (Alta Savoia) e TI-Tignes (Savoia), RI-Ribordone e CA-Carema (Piemonte). Dall'inchiesta venne chiaramente esclusa l'area walser della Valle del Lys (3 comuni; in grigio in fig. 1).

2. Per il dettaglio sulle fasi del progetto e sulla natura dei dati, nonché per una bibliografia sugli studi parziali condotti sui materiali disponibili aggiornata, si rimanda qui a Raimondi (2019).

3. Il volume Le lait et les activités laitières sarà composto da 112 voci principali incentrate sulle domande del questionario spogliate (fra Articles standard, corredati di carta analitica delle risposte, e voci provenienti da domande aperte, in cui i risultati sono presentati in forma di testi in tabella), cui si aggiungono 184 voci secondarie fra $C+$ e $R+$. I materiali sono suddivisi in cinque sezioni tematiche: I. La traite (mungitura, attrezzi e operazioni connesse), II. Entre l'étable, la cave et la fruitière (trasporto e conservazione del latte), III. Le beurre, IV. Les produits des petits-laits (lavorazione dei derivati di seconda caseificazione), V. Le fromage.

4. Da APV 1-67 le moule à beurre, CS-2 ETHN ustensiles pour mouler le beurre. La carta simbolica rivela alcune evidenze etnologiche, in particolare l'esistenza di una sorta di opposizione fra la Bassa Valle verso il Piemonte, dove sopravvivono pratiche di carattere «domestico» nella lavorazione del burro, quali l'uso del piatto concavo (generalmente detta coponnetta, rombo/ viola) o di altri contenitori simili (stella/bruno), e il resto del territorio dove questi usi non vengono riportati, segno di uno stadio più avanzato di commercializzazione. In questa seconda area, l'utilizzo della marca riportante il nome della latteria consortile (triangolo/blu) conferma questo orientamento; ad essa si oppone un'area nord-orientale dove invece è citata piuttosto la paletta (cerchio/verde) per battere e marcare il burro.

5. Per il concetto di «iconimo» come termine tecnico per designare l'associazione semanticofunzionale che permette l'utilizzo di un lessema preesistente, o di un suo derivato (segnalati convenzionalmente con le parentesi graffe), per denominare un nuovo referente, si rimanda ad Alinei (1997).

6. L'etimo per *beus è proposto da FEW I, 658-660 e ricondotto alla famiglia galloromanza dei derivati da *bŭttia «tonneau» (per il lat. class. bŭttem) come retroformazione maschile; ipotesi che viene nella CS accettata, anche se con il margine di incertezza segnalato convenzionalmente dall'uso delle parentesi quadre. Chiara invece la formazione di *burraria, come derivato a partire dalla base būt(y)ru «burro».

7. Si noti nelle figg. 7 e 8 che l'oggetto di analisi è rappresentato dal fonema (convenzionalmente, tra barre oblique) e non dal grafema che lo rappresenta nella base etimologica. Il sistema fonologico etimologico scelto come riferimento è infatti quello dello strato linguistico tradizionalmente definito «latino volgare», caratterizzato ad esempio dal passaggio del sistema vocalico latino, a dieci vocali distinte per timbro e per durata, a quello a sette vocali distinte solo 
per timbro: /i/, /e/, / $/ /, / \mathrm{a} /, / \mathrm{J} /, / \mathrm{o} /, / \mathrm{u} /$ e più modernamente battezzato «protoromanzo occidentale» (Vincent, 1988), sistema alla base anche del francoprovenzale. Questo implica che in posizione tonica, nel caso di due basi etimologiche come pŭppa e dōlŭu, il fonema oggetto di analisi sarà indicato sempre come $/ \mathrm{o} /$ tonico $(\breve{u}, \bar{o}>/ o /)$.

8. Le due sintesi (tratte da APV 1-30 écrémer e da APV 1-15 faire de la moussse en trayant, con attestazioni tratte anche da APV 1-42 faire bouillir le lait) mostrano come, anche nel caso ricorrente della tipica opposizione fra Alta Valle e Bassa Valle, l'estensione areale possa in realtà variare. Nello specifico, l'area della mancata palatalizzazione di /a/ tonica in facĕre (tratto che oppone il francoprovenzale alle varietà oiliche) mostra nell'esempio B. un'estensione verso ovest maggiore (triangolo/blu) rispetto alla conservazione di /s/ seguita da consonante, tratto fonologico caratterizzante invece per l'opposizione gallo-/italoromanza.

9. In C., tratta da APV 1-6 un trayon (du pis de la vache), si evidenzia la solidarietà dell'area rispetto alla conservazione della /a/ atona finale quando non preceduta da contesto palatale (tipo etimologico pŭppa), uno dei tratti costitutivi (Martin, 1990: 633-634; Tuaillon, 2007: 15-18) della «particolar combinazione» (secondo la celebre definizione di Ascoli, citata e discussa in Tuaillon, ibid.) che definisce il gruppo francoprovenzale. Un medesimo orientamento solidale si riscontra per un fenomeno che invece conoscerebbe realizzazioni differenti all'interno del dominio in oggetto, e cioè la palatalizzazione/conservazione di /u/ etimologica (Martin, 1990: 681; Tuaillon, 2007: 204-211); qui (esempio D.: esiti di /u/ in dūra, da APV 1-19 divers défauts des vaches à la traite, C+02 (vache qui est) difficile à traire), l'area valdostana è orientata decisamente verso la soluzione evolutiva /y/, in accordo con l'area oilica e con la maggioranza di quella francoprovenzale, con attestazione però anche della possibile «délabialisation» (Tuaillon, 2007: 207-208) in /i/ a QUQuart (quadrato/giallo). Date le caratteristiche geo-sociolinguistiche tendenzialmente innovative del punto (situato sull'asse viario principale alle porte del capoluogo regionale), i dati APV permettono di rispondere al quesito sulla natura conservativa (variante di $/ \mathrm{u} /$ etimologica) o innovativa (evoluzione ulteriore $\mathrm{di} / \mathrm{y} /$ ) dell'esito (Tuaillon, ibid.), confermando quanto è peraltro già vero dal punto di vista della fonetica naturale (/y/ è, per articolazione, fono intermedio fra / $\mathrm{u} / \mathrm{e} / \mathrm{i} / \mathrm{)}$.

10. Gli esempi sono tratti rispettivamente da APV 1-20 couler le lait (après la traite) e APV 1-33 l'écrémeuse moderne.

11. La base etimologica è qui il probabile celtismo di area galloromanza *souka "pasto» (FEW XII, 131-132), che produce il tipo lessicale *souye. Data l'inconsistenza fonologica di un eventuale passaggio diretto $/ \mathrm{k} />/ \mathrm{v} /$, l'ipotesi che quest'ultimo fonema sia il risultato di una successiva epentesi consonantica a partire da *soua (esito con dileguo attestato in tre punti d'inchiesta: triangolo/blu) rimane l'unica percorribile e consegna la forma souva di AY-Ayas alla stessa classe simbolica (triangolo/azzurro), opposta a quella maggioritaria (quadrato/arancio).

12. La motivazione principale risiede ovviamente nell'esistenza di un grado intermedio / $\mho / \mathrm{di}$ apertura (quadrato/arancio chiaro) attestato nella stessa area centrale degli altri due esiti. La moderata centralizzazione abbinata a questo grado intermedio prelude inoltre a quella più marcata dell'esito /ø/ attestato nel punto laterale di VT-Valtournenche.

13. Analoga l'impostazione nel precedente Grassi (1955-1957). Questa distinzione macro -areale trova parziale conferma nella sintesi dendrometrica in Goebl (2003: 639), basata sui dati dei soli tre punti valdostani dell'AIS (Rhêmes-Notre-Dame e Saint-Marcel, in Alta e Media Valle, di contro a Brusson nella Bassa Valle).

14. In merito, rimando alle osservazioni quanti-qualitative compiute da chi scrive sulla caratterizzazione del lessico del francoprovenzale valdostano in rapporto piuttosto alle macroaree galloromanza e italoromanza settentrionale (Raimondi, 2016).

15. Per una rassegna piuttosto esaustiva dei due autori si vedano le bibliografie contenute sul sito del Dialektometrie Projekt-Salzburg (<www.dialectometry.com/index.php>), nelle diverse sezioni Ladinia, Frankreich, Toscana, Italien. 
16. Il campione è stato definito statisticamente sulla base della stabilizzazione progressiva del rango dei punti d'inchiesta nella graduatoria di similarità con il punto di riferimento SA-Sarre, arrestandosi quando esso non subiva più variazioni significative ( $\Delta$ Ranking $\leq 1$ progredendo nel campionamento a gruppi di 10 voci). Nel passaggio dalle 40 alle 50 voci spogliate, 13 dei 22 punti d'inchiesta risultavano stabili, tutti rispettavano il parametro $\Delta$ Ranking. Dall'analisi sono state ovviamente escluse le sintesi nelle quali il punto di Sarre non conteneva risposte.

17. Tecnicamente, il colore è stato determinato agendo sul solo valore $\mathrm{H}$ (Hue, «tonalità») del sistema di composizione digitale HSV (Hue, Saturation, Brightness, «tonalità, saturazione, luminosità»), attribuendogli un valore compreso fra 0 (rosso, corrispondente a \%SIM $=100$ ) e 89 (verde limone, $\% \mathrm{SIM}_{\mathrm{M}}=0$ ). Il cerchio è stato adottato qui come soluzione alternativa alla poligonazione di scuola salisburghese.

18. Nel metodo seguito, l'aumento della convergenza è un'ovvia conseguenza dell'automatica esclusione dal computo delle sintesi linguistiche che sono "globalmente divergenti», in cui i dati presentano cioè un'estrema variazione quasi punto per punto.

\section{RIASSUNTI}

Accanto a una breve illustrazione della storia del progetto di questo atlante linguistico regionale e della struttura informativa del suo primo volume in corso di pubblicazione, il contributo propone un approfondimento sul tema della cartografia sintetica utilizzata e delle metodologie seguite per costruirla ed offre poi un saggio di lettura dialettometrica di un campione dei suoi dati linguistici e, alla luce di questo, alcune chiavi di lettura delle dinamiche che spiegano la configurazione geolinguistica piuttosto complessa del territorio valdostano.

Parallèlement à un bref historique du projet de cet atlas linguistique régional et d'une illustration de la structure informative de son premier volume (à paraître), l'article développe le sujet de la cartographie synthétique utilisée et détaille la méthodologie suivie pour la réaliser, en proposant enfin un essai d'interprétation dialectométrique d'un échantillon de ses données linguistiques. Sur cette base, quelques clés de lecture des dynamiques capables d'expliquer la configuration géolinguistique très complexe du territoire valdôtain sont aussi introduites.

After a short history of the project of this regional linguistic atlas and an overview on the informational structure of its first volume (published soon), this paper offers an in-depth examination of the synthetical maps' methodologies used to implement it. Subsequently, it gives an example of dialectometric applications on a sample of APV's data and, in the light of these, it propose some keys to understanding the dynamics that can explain the fairly complex geolinguistic landscape of Aosta Valley.

\section{INDICE}

Parole chiave : Valle d'Aosta, francoprovenzale, atlante linguistico, dialettometria

Keywords : Valle d'Aosta, Francoprovençal, linguistic atlas, dialectométry

Mots-clés : Val d'Aoste, francoprovençal, atlas linguistique, dialectométrie 


\section{AUTORE}

\section{GIANMARIO RAIMONDI}

Università della Valle d'Aosta / Université de la Vallée d'Aoste

g.raimondi@univda.it 